\title{
Plant Cytogenetics, 3rd edition
}

\author{
Ram J. Singh, CRC Press, November 2016, 528 Pages - 143 Illustrations, Print ISBN: \\ 978-1-4398-8418-8, eBook ISBN: 978-1-4398-8419-5, DOI: 10.1201/9781315374611, \\ Hardback Print $£$ 82, ebook $£ 57$
}

\author{
Umesh C. Lavania ${ }^{1}$
}

Published online: 16 March 2017

(c) Archana Sharma Foundation of Calcutta 2017

Rediscovery of Mendelian Laws of Inheritance generated interest in understanding chromosomal basis of inheritance. Therefore, experimental procedures and techniques were developed to elucidate structure of chromosomes and understand their behavior to underscore genetic principles. The hybrid discipline that combines cytology and genetics is termed as cytogenetics. The author himself an accomplished plant cytogeneticist has taken the challenge to provide an exhaustive account of the subject area in the form of this book. Over the past two decades there has been vast technical advancement in this area. Therefore, it is desirable to bring out an updated account commensurate with the progressive development. Author has aptly done this in this 3rd edition, by providing holistic treatment covering classical and modern techniques of plant cytogenetics, and their application in chromosomal/cytogenetic analysis and plant breeding.

This updated edition runs into 12 Chapters, beginning with Introduction of the subject area tracking its development from Mendelian Genetics to Chromosomal basis of inheritance; followed by Handling of Plant Chromosomes giving details of protocols, Cell Division covering mitotic and meiotic analysis, including differences in the two division processes; Genetic control of meiosis from its occurrence and utilitarian viewpoint; Mode of Reproduction in Plants; Karyotype analysis; Chromosome aberrations, including origin and identification of translocations, duplications, deficiencies, polyploidy and aneuploidy and

Umesh C. Lavania

lavaniauc@yahoo.co.in

1 Department of Botany, University of Lucknow, Lucknow 226007, India addition lines in genetic studies; Genome analysis; Cytogenetic aberrations in Tissue Culture; Transgenic Crops; and Relationship between Cytogenetics and Plant Breeding covering recombination, mutations, polyploidy, genetic transformation, and wide hybridization.

Whereas, molecular cytogenetics and genomics have been vastly enriched by the availability of cytogenetic chromosome maps in barley, tomato, rice, maize and wheat that were earlier developed by pioneering cytogeneticists using aneuploid stocks, but necessary interest in this area is witnessing a declining trend for want of trained personnel in plant cytogenetics. Therefore, availability of the updated and exhaustive account of the subject area in the form of this book is timely. This book may serve as a fillip to rejuvenate interest in the study of plant cytogenetics and help develop trained people in this important area both in the developing and developed economies. However, in order to make increased availability of this book within the reach of individual students from developing countries, it would be appreciated if a paperback edition at a reduced price is also published.

All in all it is an appreciable effort by the author to bring out an updated treatment of the subject area, since all other available books on related subject are quite old lacking recent advances. I believe that the students and teachers interested in understanding and applying plant cytogenetics would find this book worth keeping on their shelf. 